\title{
High PD-L1 Expression Associates with Low T- Cadherin Expression and Poor Prognosis in HPV- Negative Head and Neck Squamous Cell Carcinoma
}

\section{Qiuju Wang}

Sichuan Cancer Hospital \& Institute https://orcid.org/0000-0001-9781-0138

\section{Yanzhen Zhao}

Third Affiliated Hospital of Guangzhou Medical College

\section{Yan Chen}

Sichuan Cancer Hospital and Research Institute: Sichuan Cancer Hospital and Institute Yibo Chen

Sichuan Cancer Hospital and Research Institute: Sichuan Cancer Hospital and Institute

\section{Xiaoyu Song}

Sichuan Cancer Hospital and Research Institute: Sichuan Cancer Hospital and Institute

\section{Li Zhang}

Sichuan Cancer Hospital and Research Institute: Sichuan Cancer Hospital and Institute

Qiao He

Sichuan Cancer Hospital and Research Institute: Sichuan Cancer Hospital and Institute

\section{Bo Ye}

Sichuan Cancer Hospital and Research Institute: Sichuan Cancer Hospital and Institute

\section{Lichun Wu}

Sichuan Cancer Hospital and Research Institute: Sichuan Cancer Hospital and Institute

\section{Xinyue Huang}

Sichuan Cancer Hospital and Research Institute: Sichuan Cancer Hospital and Institute

\section{Dongsheng Wang (D864392782@qq.com)}

Sichuan Cancer Hospital and Research Institute: Sichuan Cancer Hospital and Institute

\section{Research Article}

Keywords: PD-L1, T-cadherin, prognosis, HPV-negative, HNSCC

Posted Date: July 7th, 2021

DOI: https://doi.org/10.21203/rs.3.rs-668097/v1

License: (1) This work is licensed under a Creative Commons Attribution 4.0 International License. Read Full License 


\section{Abstract}

Purpose

T-cadherin is an immunoglobulin-like adhesion molecule which acts as a tumor suppressor gene, programmed cell death ligand 1 (PD-L1) is a cell surface protein that involves in the suppression of the immune system. This study aimed at exploring the correlation between T-cadherin and PD-L1, as well as their prognostic value in patients with HPV-negative head and neck squamous cell carcinoma (HNSCC).

Methods

In this study, immunohistochemical staining was used to determine the protein expression of T-cadherin and PD-L1 in 104 tissue specimens of HPV-negative HNSCC. Spearman linear correlation analysis was used to determine the association between protein expression of T-cadherin and PD-L1. Kaplan-Meier analysis was used to plot overall survival (OS) and disease-free survival (DFS) curves. Cox proportional hazards regression analysis was used to conduct univariate and multivariate analysis.

Results

The results showed a large negative association between protein expression of T-cadherin and PD-L1 $(r=-0.775$, $P<0.01)$, expression of T-cadherin and PD-L1 were associated with OS ( $P=0.021$ and 0.034 , respectively) and DFS ( $P=0.012$ and 0.016 , respectively) in patients with HPV-negative HNSCC. Cox proportional hazards regression analysis revealed that expression of T-cadherin and PD-L1 were independent prognostic predictors for OS and DFS in patients with HPV-negative HNSCC. The worst prognosis was observed in patients with Tcadherin negative/PD-L1 positive.

Conclusion

In conclusion, expression of T-cadherin and PD-L1 were inversely correlated and were independent prognostic factors for patients with HPV-negative HNSCC.

\section{Introduction}

Head and neck squamous cell carcinoma (HNSCC) is the sixth most common malignancy worldwide (Alsahafi et al. 2017), with approximately 650,000 new cases reported each year (Ferlay et al. 2015). Despite advances in both diagnosis and therapy in recent decades, the five-year survival rate of HNSCC is still low, resulting in more than 140,000 deaths annually (Ang et al. 2014). The low survival rate might be due to late diagnosis and a lack of biomarkers that can effectively predict the progression and prognosis of HNSCC (Leemans et al. 2011). Thus, identifying effective biomarkers would be of great importance for the treatment and early prognosis prediction of patients with HNSCC.

T-cadherin is a member of the cadherin superfamily that belongs to intercellular adhesion molecules (Philippova et al. 2013). The expression of T-cadherin is reduced or lost in various types of cancer (Philippova et al. 2013; Abudukadeer et al. 2012; Duan et al. 2013), while its overexpression can significantly inhibit the proliferation and invasion of various tumor cells (Huang et al.2003; Mukoyama et al. 2005; Kyriakakis et al. 
2012). Our previous study demonstrated that the expression of T-cadherin is associated with a poor prognosis of oral squamous cell carcinoma (Wang et al. 2018). These studies (Philippova et al. 2013; Abudukadeer et al. 2012; Duan et al. 2013; Huang et al.2003; Mukoyama et al. 2005; Kyriakakis et al. 2012; Wang et al. 2018) indicate that T-cadherin plays an important role as a tumor suppressor gene and may act as a potential target for cancer therapy. Programmed death-ligand 1(PD-L1) is a cell-surface protein that belongs to the B7 superfamily. PD-L1 interacts with the programmed cell death protein 1 (PD-1) and forms an important cancer immune escape mechanism (Cohen et al. 2019). Previous studies have found that E-cadherin and N-cadherin, other members of the cadherin family, have a certain regulatory relationship with PD-L1(Suda et al. 2017; Sun et al. 2021). However, there has not been any study on the correlation between T-cadherin and PD-L1, so this study is designed to explore it. Furthermore, current studies on the correlation between PD-L1 expression and the prognosis for cancer are inconsistent (Ohigashi et al. 2005; Thompson et al. 2004; Ferrand et al. 2013; Evrard et al. 2020), these contradictory results prompted us to further investigate the relationship between the expression of PD-L1 and the prognosis in patients with HNSCC. Thus, the current study aimed to explore the expression of T-cadherin and PD-L1, as well as their association in patients with HNSCC. Furthermore, the prognostic value of T-cadherin and PD-L1, alone or in combination, was also investigated.

\section{Materials And Methods}

\section{Patients}

A total of 104 patients who received radical surgery between April 2017 and August 2021 at Sichuan Cancer Hospital \& Institute were included in this study. The inclusion criteria were as follows: patients with HNSCC were pathologically confirmed, patients can provide sufficient tissues to meet the requirements of the study, patients with HPV DNA negative and P16 negative, patients are followed up and are willing to join the study. Exclusion criteria were patients with any other malignant disease, patients with distant metastasis, patients with HPV DNA positive or P16 positive, patients who have received radiation or chemotherapy prior to surgery, patients with follow-up loss or death not associated with tumor progression. Patients were staged according to the eighth edition of American Joint Committee on Cancer (AJCC) staging system for head and neck cancer. The study was approved by the ethics committee of Sichuan Cancer Hospital \& Institute (Approval Number: SCCHEC-02-2019-016), written informed consent was obtained from each patient.

\section{Immunohistochemical (IHC) staining of T-cadherin and PD-L1}

104 formalin-fixed paraffin-embedded tissue specimens of HNSCC were sectioned at 4 um, deparaffinized in xylene after heat drying, then heat-induced epitope retrieval was conducted using heated citrate buffer for 20 min, endogenous peroxidase was blocked by $3 \%$ hydrogen peroxide, slides were incubated at $4{ }^{\circ} \mathrm{C}$ overnight with rabbit monoclonal primary PD-L1 antibody (No. 13684S, clone E1L3N, 1:200 dilution, Cell Signaling Technology, Danvers, MA, USA) and rabbit monoclonal to T-cadherin antibody (ab167407, EPR9621, 1:200 dilution, Abcam, Cambrige, UK). Anti-rabbit horseradish peroxidase (HRP)-conjugated secondary antibody was added to the slides at room temperature for 30 minutes after washing, and 3,3'-diaminobenzidine (DAB) was used to stain the slides and haematoxylin was used for counterstaining. Each slide was scored by two pathologists independently. Tumor proportion score (TPS) was defined as the percentage of viable tumor cells showing partial or complete membrane staining at any intensity. Tumor cells with TPS $\geq 5$ positive localization 
of PD-L1 in the membrane were considered positive, and tumor cells with TPS $₫ 1$ positive localization of Tcadherin in the membrane were considered positive.

\section{Statistical analysis}

Statistical analysis was performed using SPSS 24.0 (IBM CorP, Armonk, NY, USA). Mann-Whitney U test was used for continuous variables and Chi-square test was used for categorical variables. The correlation between expression of T-cadherin and PD-L1 was analyzed by spearman's correlation analysis. Kaplan-Meier survival analysis was used to plot survival curves and log-rank test was used to determine the significance. Univariate and multivariate analyses were performed by cox proportional hazards regression analysis. $\mathrm{P}<0.05$ was considered a statistically significant difference.

\section{Results}

\section{Demographic and clinicopathological characteristics of patients}

The demographic and clinical characteristics of 104 HPV-negative HNSCC (HPV DNA-negative, P16-negative) are shown in Table 1. The majority of patients were men $(78,75.0 \%)$ and from non-laryngopharynx $(88,84.6 \%)$, the median age was 62 years (range from 25 to 79 years). 82 (78.8\%) patients had used tobacco and 54 (51.9\%) consumed alcohol, the median follow-up time was 32 months. 
Table 1

Demographic and clinicopathological characteristics of the study patients by T-cadherin and PD-L1 status

\begin{tabular}{|c|c|c|c|c|c|c|c|}
\hline Characteristic & $\begin{array}{l}\text { All } \\
\text { patients(N } \\
=104)\end{array}$ & $\begin{array}{l}\text { T-cadherin } \\
\text { positive( } N \\
=14)\end{array}$ & $\begin{array}{l}\text { T-cadherin } \\
\text { negative }(\mathrm{N} \\
=90)\end{array}$ & $\mathbf{P}$ & $\begin{array}{l}\text { PD-L1 } \\
\text { positive(N } \\
=67)\end{array}$ & $\begin{array}{l}\text { PD-L1 } \\
\text { negative(N } \\
=37)\end{array}$ & $P$ \\
\hline \multicolumn{8}{|l|}{ Gender } \\
\hline Male & $78(75.0 \%)$ & $10(71.4 \%)$ & $68(75.6 \%)$ & 0.74 & $46(68.7 \%)$ & $32(86.5 \%)$ & $0.044^{*}$ \\
\hline Female & $26(25.0 \%)$ & $4(28.6 \%)$ & $22(24.4 \%)$ & & $21(31.3 \%)$ & $5(13.5 \%)$ & \\
\hline \multicolumn{8}{|l|}{ Age } \\
\hline$<60$ & $38(36.5 \%)$ & $5(35.7 \%)$ & $33(36.7 \%)$ & 0.945 & $22(32.8 \%)$ & $16(43.2 \%)$ & 0.291 \\
\hline$\geq 60$ & $66(63.5 \%)$ & $9(64.3 \%)$ & $57(63.3 \%)$ & & $45(67.2 \%)$ & $21(56.8 \%)$ & \\
\hline \multicolumn{8}{|l|}{ T stage } \\
\hline $\mathrm{T} 1-2$ & $54(51.9 \%)$ & $12(85.7 \%)$ & $42(46.7 \%)$ & $0.015^{*}$ & $27(40.3 \%)$ & $27(73.0 \%)$ & $0.001^{*}$ \\
\hline T3-4 & $50(48.1 \%)$ & $2(14.3 \%)$ & $48(53.3 \%)$ & & $40(59.7 \%)$ & $10(27.0 \%)$ & \\
\hline \multicolumn{8}{|l|}{$\mathrm{N}$ stage } \\
\hline NO & $42(40.4 \%)$ & $2(14.3 \%)$ & $40(44.4 \%)$ & $0.032^{*}$ & $23(34.3 \%)$ & $22(59.5 \%)$ & $0.013^{*}$ \\
\hline N1-3 & $62(59.6 \%)$ & $12(85.7 \%)$ & $50(55.6 \%)$ & & $44(59 . \%)$ & $15(40.5 \%)$ & \\
\hline \multicolumn{8}{|l|}{ Smoking } \\
\hline No & $22(21.2 \%)$ & $5(35.7 \%)$ & $17(18.9 \%)$ & 0.152 & $16(23.9 \%)$ & $6(16.2 \%)$ & 0.36 \\
\hline Yes & $82(78.8 \%)$ & $9(64.3 \%)$ & 73(81.1\%) & & $51(76.1 \%)$ & $31(83.8 \%)$ & \\
\hline \multicolumn{8}{|l|}{ Drinking } \\
\hline No & $50(48.1 \%)$ & $6(42.9 \%)$ & $44(48.9 \%)$ & 0.674 & $33(49.3 \%)$ & $17(45.9 \%)$ & 0.747 \\
\hline Yes & $54(51.9 \%)$ & $8(57.1 \%)$ & $46(51.1 \%)$ & & $34(50.7 \%)$ & $20(54.1 \%)$ & \\
\hline \multicolumn{8}{|l|}{ Sites } \\
\hline $\begin{array}{l}\text { Non- } \\
\text { laryngopharynx }\end{array}$ & $88(84.6 \%)$ & $10(71.4 \%)$ & $78(86.7 \%)$ & 0.142 & $58(86.6 \%)$ & $30(81.1 \%)$ & 0.458 \\
\hline Laryngopharynx & $16(15.4 \%)$ & $4(28.6 \%)$ & $12(13.3 \%)$ & & $9(13.4 \%)$ & $7(18.9 \%)$ & \\
\hline \multicolumn{8}{|l|}{$\begin{array}{l}\text { T-cadherin } \\
\text { status }\end{array}$} \\
\hline Positive & $14(13.5 \%)$ & & & & $3(4.5 \%)$ & $11(29.7 \%)$ & $<.001^{*}$ \\
\hline Negative & $90(86.5 \%)$ & & & & $64(95.5 \%)$ & $26(70.3 \%)$ & \\
\hline
\end{tabular}

${ }^{*} \mathrm{P}<0.05$; Radio, radiotherapy; Chemo, Chemotherapy; PD-L1, programmed cell death 1 ligand 1. 


\begin{tabular}{|c|c|c|c|c|c|c|c|}
\hline Characteristic & $\begin{array}{l}\text { All } \\
\text { patients(N } \\
=104)\end{array}$ & $\begin{array}{l}\text { T-cadherin } \\
\text { positive(N } \\
=14)\end{array}$ & $\begin{array}{l}\text { T-cadherin } \\
\text { negative(N } \\
=90)\end{array}$ & $\mathbf{P}$ & $\begin{array}{l}\text { PD-L1 } \\
\text { positive(N } \\
=67)\end{array}$ & $\begin{array}{l}\text { PD-L1 } \\
\text { negative(N } \\
=37)\end{array}$ & $\mathbf{P}$ \\
\hline \multicolumn{8}{|l|}{ PD-L1 status } \\
\hline Positive & $67(64.4 \%)$ & $2(14.3 \%)$ & $65(72.2 \%)$ & $\begin{array}{l}< \\
0.001^{*}\end{array}$ & & & \\
\hline Negative & $37(35.6 \%)$ & $12(85.7 \%)$ & $25(27.8 \%)$ & & & & \\
\hline
\end{tabular}

\section{Association of T-cadherin or PD-L1 expression with various demographic and clinicopathological characteristics of HPV-negative HNSCC.}

The representative expression of T-cadherin and PD-L1 are shown in Fig. 1. Among the 104 patients with HPVnegative HNSCC, 90 (86.5\%) samples exhibited T-cadherin negative, low expression of T-cadherin was significantly associated with advanced T stage $(P=0.015)$, N-stage $(P=0.032)$, and high expression of PD-L1 $(P<0.001)$, but not with gender, age, smoking status, drinking status or tumor sites. 67 (64.4\%) samples presented PD-L1 positive, high expression of PD-L1 was significantly correlated with gender $(P=0.044)$, advanced T-stage $(P=0.001)$, N-stage $(P=0.013)$, and low expression of T-cadherin $(P<0.001)$, but not with age, smoking status, drinking status or tumor sites (Table 1). Spearman linear correlation analysis demonstrated a large negative linear correlation between protein expression of T-cadherin and PD-L1 in the same patient ( $r=-0.775, P<0.01$, Fig. 2).

\section{Low T-cadherin expression and high PD-L1 expression are associated with poor prognosis of HPV-negative HNSCC}

Kaplan-Meier analysis revealed that low T-cadherin or high PD-L1 expression tend to have shorter OS ( $P=0.021$ and 0.034 , respectively) and DFS ( $P=0.012$ and 0.016 , respectively, Fig. 3$)$. Univariate analysis showed that gender, T-stage, N-stage, expression levels of T-cadherin and PD-L1 were associated with both OS $(P<0.05$, Table 2) and DFS $(P<0.05$, Table 3$)$ of patients with HPV-negative HNSCC. Multivariate analysis demonstrated that T-stage, $\mathrm{N}$-stage, expression levels of T-cadherin and PD-L1 were found as independent predictors for shorter OS $(P<0.05$, Table 2$)$ and DFS $(P<0.05$, Table 3$)$ of patients with HPV-negative HNSCC. 
Table 2

Univariate and multivariate analysis for overall survival (OS) of HNSCC patients.

\begin{tabular}{|c|c|c|c|c|}
\hline \multirow[t]{2}{*}{ Characteristic } & \multicolumn{2}{|c|}{ OS univariate analysis } & \multicolumn{2}{|c|}{ OS multivariate analysis } \\
\hline & $\mathrm{HR}(95 \% \mathrm{Cl})$ & P-value & $\mathrm{HR}(95 \% \mathrm{Cl})$ & P-value \\
\hline \multicolumn{5}{|l|}{ Gender } \\
\hline Female & 1 & & 1 & \\
\hline Male & $2.68(1.14,3.02)$ & $0.042^{*}$ & $1.86(1.28,2.84)$ & 0.162 \\
\hline \multicolumn{5}{|c|}{ Age at diagnosis } \\
\hline$<60$ & 1 & & & \\
\hline$\geq 60$ & $1.56(0.76,2.32)$ & 0.246 & & \\
\hline \multicolumn{5}{|l|}{ Smoking } \\
\hline No & 1 & & & \\
\hline Yes & $1.12(0.52,1.78)$ & 0.548 & & \\
\hline \multicolumn{5}{|l|}{ Drinking } \\
\hline No & 1 & & & \\
\hline Yes & $1.24(0.64,1.89)$ & 0.425 & & \\
\hline \multicolumn{5}{|l|}{ T stage } \\
\hline T1-2 & 1 & & 1 & \\
\hline T3-4 & $3.24(1.56,4.88)$ & $0.002^{\star}$ & $3.04(1.48,5.06)$ & $0.004^{*}$ \\
\hline \multicolumn{5}{|l|}{ N stage } \\
\hline NO & 1 & & 1 & \\
\hline N1-3 & $3.86(1.45,4.26)$ & $0.001^{*}$ & $2.96(1.52,3.86)$ & $0.003^{*}$ \\
\hline \multicolumn{5}{|c|}{ T-cadherin status } \\
\hline Positive & 1 & & 1 & \\
\hline Negative & $4.68(2.11,7.56)$ & $\varangle 0.001^{*}$ & $3.92(1.98,6.57)$ & $0.001^{*}$ \\
\hline \multicolumn{5}{|l|}{ PD-L1 status } \\
\hline Negative & 1 & & 1 & \\
\hline Positive & $6.48(2.42,9.58)$ & $\otimes 0.001^{*}$ & $4.98(2.56,8.76)$ & $\varangle 0.001^{*}$ \\
\hline
\end{tabular}


Table 3

Univariate and multivariate analysis for deasease-free survival (DFS) of HNSCC patients.

\begin{tabular}{|c|c|c|c|c|}
\hline \multirow[t]{2}{*}{ Characteristic } & \multicolumn{2}{|c|}{ DFS univariate analysis } & \multicolumn{2}{|c|}{ DFS multivariate analysis } \\
\hline & $\mathrm{HR}(95 \% \mathrm{Cl})$ & P-value & $\mathrm{HR}(95 \% \mathrm{Cl})$ & P-value \\
\hline \multicolumn{5}{|l|}{ Gender } \\
\hline Female & 1 & & 1 & \\
\hline Male & $3.02(1.12,3.42)$ & 0.036 & $1.88(0.56-3.12)$ & 0.125 \\
\hline \multicolumn{5}{|c|}{ Age at diagnosis } \\
\hline$<60$ & 1 & & & \\
\hline$\geq 60$ & $1.66(0.76,2.62)$ & 0.262 & & \\
\hline \multicolumn{5}{|l|}{ Smoking } \\
\hline No & 1 & & & \\
\hline Yes & $1.82(0.84,2.96)$ & 0.138 & & \\
\hline \multicolumn{5}{|l|}{ Drinking } \\
\hline No & 1 & & & \\
\hline Yes & $1.64(0.66,2.58)$ & 0.325 & & \\
\hline \multicolumn{5}{|l|}{ T stage } \\
\hline T1-2 & 1 & & 1 & \\
\hline T3-4 & $3.12(1.46,5.28)$ & $0.003^{*}$ & $2.88(1.38,4.84)$ & $0.004^{*}$ \\
\hline \multicolumn{5}{|l|}{ N stage } \\
\hline No & 1 & & 1 & \\
\hline N1-3 & $3.82(1.28,5.16)$ & $0.001^{*}$ & $3.48(1.52,4.96)$ & $0.002^{\star}$ \\
\hline \multicolumn{5}{|c|}{ T-cadherin status } \\
\hline Positive & 1 & & 1 & \\
\hline Negative & $5.22(2.46,8.48)$ & $\nabla 0.001^{*}$ & $4.89(1.99,7.45)$ & $\nabla 0.001^{*}$ \\
\hline \multicolumn{5}{|l|}{ PD-L1 status } \\
\hline Negative & 1 & & 1 & \\
\hline Positive & $6.64(2.52,11.58)$ & $\bowtie 0.001^{*}$ & $5.32(2.32,10.74)$ & $\bowtie 0.001^{*}$ \\
\hline
\end{tabular}


Kaplan-Meier analysis showed that there was a significant difference on OS and DFS of HPV-negative HNSCC by expression of T-cadherin and PD-L1 combination $(P<0.001$, Fig. 4). The best prognosis was observed in those patients with T-cadherin positive/PD-L1 negative and the worst prognosis were seen in patients with Tcadherin negative/PD-L1 positve (Tables 4 and 5). The OS of patients with T-cadherin negative/PD-L1 positve were 8.16 times shorter than patients with T-cadherin positive/PD-L1 negative $(P<0.001, T a b l e .4)$, the DFS of patients with T-cadherin negative/PD-L1 positve were 9.28 times shorter than patients with T-cadherin positive/ PD-L1 negative $(P<0.001$, Table. 5).

Table 4

Univariate and multivariate analysis of the prognostic value of T-cadherin in combination with PD-L1 expression regarding OS in patients with HNSCC

\begin{tabular}{|c|c|c|c|c|}
\hline \multirow[t]{2}{*}{ T-cadherin and PD-L1 status } & \multicolumn{2}{|c|}{ OS univariate analysis } & \multicolumn{2}{|c|}{ OS multivariate analysis } \\
\hline & $\mathrm{HR}(95 \% \mathrm{Cl})$ & P-value & $\mathrm{HR}(95 \% \mathrm{Cl})$ & P-value \\
\hline T-cadherin(+) and PD-L1(-) & 1 & & 1 & \\
\hline T-cadherin(-) and PD-L1(-) & $2.62(0.82,4.26)$ & 0.028 & $3.26(1.08,5.32)$ & 0.008 \\
\hline T-cadherin(+) and PD-L1(+) & $5.68(1.12,8.25)$ & $\bowtie 0.001$ & $6.98(1.92,9.66)$ & $\bowtie 0.001$ \\
\hline T-cadherin(-) and PD-L1(+) & $7.32(1.38,11.56)$ & $\varangle 0.001$ & $8.16(2.02,13.22)$ & $凶 0.001$ \\
\hline
\end{tabular}

Table 5

Univariate and multivariate analysis of the prognostic value of T-cadherin in combination with PD-L1 expression regarding DFS in patients with HNSCC

\begin{tabular}{|c|c|c|c|c|}
\hline \multirow[t]{2}{*}{ T-cadherin and PD-L1 status } & \multicolumn{2}{|c|}{ DFS univariate analysis } & \multicolumn{2}{|c|}{ DFS multivariate analysis } \\
\hline & $\mathrm{HR}(95 \% \mathrm{Cl})$ & P-value & $\mathrm{HR}(95 \% \mathrm{Cl})$ & P-value \\
\hline T-cadherin(+) and PD-L1(-) & 1 & & 1 & \\
\hline T-cadherin(-) and PD-L1(-) & $3.18(1.26,5.24)$ & 0.014 & $3.92(1.38,6.11)$ & 0.006 \\
\hline T-cadherin(+) and PD-L1(+) & $6.16(1.08,9.82)$ & $\nabla 0.001$ & $7.14(1.86,10.66)$ & $\varangle 0.001$ \\
\hline T-cadherin(-) and PD-L1(+) & $8.12(1.58,12.88)$ & $\bowtie 0.001$ & $9.28(2.46,14.62)$ & $\bowtie 0.001$ \\
\hline
\end{tabular}

\section{Discussion}

The main causes of HPV-negative HNSCC are patients' tobacco and alcohol consumption. Patients consuming tobacco and alcohol have been reported to respond worse to therapy and have worse outcomes (O'Rorke et al. 2012). The current study demonstrates a reduced expression of T-cadherin in the tissues of HPV-negative HNSCC patients. In accordance with our previous study, which revealed a low expression of T-cadherin is associated with advanced stage of patients with HNSCC (Wang et al. 2018). Our results show a 64.4\% positive PD-L1 expression in HPV-negative HNSCC; however, the expression of PD-L1 has varied in other studies (ranging from 29-100\%) (Bauml et al. 2017; Burtness et al. 2019; Seiwert et al. 2016). The variation in the level of PD-L1 expression can be attributed to different antibodies used for staining, the heterogeneity of samples, fresh or paraffin-embedded samples, PD-L1 expression on inflammatory cells being scored or not, and different cutoffs (Cho et al. 2011). 
The present study shows that a high expression of PD-L1 is correlated with gender, stage, and different treatment options, which is consistent with the study performed on oropharyngeal cancer (Hong et al. 2019). Our study finds that the expression of T-cadherin is negatively correlated with the expression of PD-L1. It has been demonstrated that the increased activation of PI3K/Akt signaling and the up-regulated expression of PDL1 are found in radiation-resistant HPV-negative cell lines (Ski Skinner et al. 2017). Our previous study found that T-cadherin can inhibit the proliferation of oral squamous cell carcinoma through a PI3K/Akt/mTOR pathway (Wang et al. 2018), these studies indicate that there may be some regulatory relationship between Tcadherin and PD-L1. Our study confirmed that the protein expression of the two genes is closely related, but the exact regulatory mechanism remains to be further studied.

Until now, the association between the expression of PD-L1 and the prognosis of HNSCC has remained controversial. Our study demonstrates that the positive expression of PD-L1 is associated with worse OS and PFS in patients with HNSCC, which is supported by studies conducted by Schneider et al (Schneider et al. 2018). and Zhou et al (2020). However, another study showed no correlation of survival with PD-L1 expression in oral squamous cell carcinoma (Chen et al. 2018). Hong et al. (2016) even found PD-L1 to be a positive prognostic factor for OS in 99 tonsillar cancer patients by univariate analysis, but this has not been the case in multivariate analysis (Chan et al. 2008). Such discrepancies may be caused by the use of different cutoffs, the heterogeneity of tumor sites, stages, treatment options. Our study used different sites of HNSCC with different treatment options and adopted the cut-off value that most studies have used (TPS $=5$ ). Thus, the findings are relatively reliable. Nevertheless, the study needs to be validated by a larger sample size and multi-center studies, and the antibody and cutoff value to be used should be standardized.

The results showed that either the expression of T-cadherin or PD-L1 is an independent prognostic factor for the OS and DFS of patients with HPV-negative HNSCC, and that patients who were T-cadherin negative and PDL1 positive had the worst prognosis. It was found that the expression of T-cadherin was reduced or lost by hypermethylation in various cancers (Chan et al. 2008; Ulivi et al. 2006) and that the hypermethylation of Tcadherin was correlated with worse prognosis in patients with cervical cancer (Widschwendter et al. 2004) and oral squamous cell carcinoma (Wang et al. 2021). It was speculated that T-cadherin and PD-L1 affect the prognosis of HPV negative HNSCC patients through certain mechanisms, while the exact mechanism why patients with T-cadherin negative/PD-L1 positive had the worst prognosis remains to be further studied.

In conclusion, the current study demonstrates that the expression of T-cadherin and PD-L1 are of large negative correlation; the low expression of T-cadherin and the high expression of PD-L1 are independent prognostic factors for worse outcomes in patients with HPV-negative HNSCC; patients with T-cadherin negative/PD-L1 positive had the worst prognosis, indicating that those patients require further personalized therapy.

\section{Declarations}

\section{Funding}

This study was supported by the healthy department of Sichuan Province (grant/award number: 20PJ110, 20PJ116); the Sichuan Provincial Cadre Health Research Project (grant/award number: 2019-802); Research Project of Sichuan Administration of Traditional Chinese Medicine (grant/award number:2020JC0114); Youth Innovation Research Project of Sichuan Medical Association (grant/award number:Q20058); Youth Scientific 
research Project of the Third Affiliated Hospital of Guangzhou Medical University (grant/award number: 2018Q4).

\section{Authors' contributions}

Qiuju Wang and Yanzhen Zhao: Conceptualization and design of the study, quality control of data and algorithms, statistical analysis, preparation, editing and review of the manuscript. Dongsheng Wang: Conceptualization and design of the study, editing and review of the manuscript. Yan Chen, Yibo Chen, Xiaoyu Song and Li Zhang: Design of the study, data analysis and interpretation, statistical analysis. Bo Ye, Lichun Wu and Xinyue Huang: Acquisition, analysis and interpretation of the data.

\section{Declaration of competing interest}

The authors declare no conflict of interests.

\section{Data availability}

The data that support the findings of this study are available from the corresponding author upon reasonable request.

Code availability Not applicable.

\section{References}

1. Abudukadeer A, Bakry R, Goebel G et al (2012) Clinical relevance of CDH1 and CDH13 DNA-methylation in serum of cervical cancer patients. Int J Mol Sci 13:8353-8363. https://doi.org/ 10.3390/ijms13078353

2. Alsahafi E, Begg K, Amelio I et al (2019) Clinical update on head and neck cancer: molecular biology and ongoing challenges. Cell Death Dis 10:540. https://doi.org/10.1038/s41419-019-1769-9

3. Ang KK, Zhang Q, Rosenthal DI et al (2014) Randomized phase III trial of concurrent accelerated radiation plus cisplatin with or without cetuximab for stage III to IV head and neck carcinoma: RTOG 0522. J Clin Oncol 32:2940-2950. https://doi.org/10.1200/JC0.2013.53.5633

4. Bauml J, Seiwert TY, Pfister DG et al (2017) Pembrolizumab for Platinum- and Cetuximab-Refractory Head and Neck Cancer: Results From a Single-Arm, Phase II Study. J Clin Oncol 35:1542-1549. https://doi.org/10.1200/JC0.2016.70.1524

5. Burtness B, Harrington KJ, Greil R et al (2019) Pembrolizumab alone or with chemotherapy versus cetuximab with chemotherapy for recurrent or metastatic squamous cell carcinoma of the head and neck (KEYNOTE-048): a randomised, open-label, phase 3 study. Lancet 394:1915-1928. https://doi.org/10.1016/S0140-6736(19)32591-7

6. Chan DW, Lee JM, Chan PC et al (2008) Genetic and epigenetic inactivation of T-cadherin in human hepatocellular carcinoma cells. Int J Cancer 123:1043-1052. https://doi.org/10.1002/ijc.23634

7. Chen SC, Chang PM, Wang HJ et al (2018) PD-L1 expression is associated with p16 INK4A expression in non-oropharyngeal head and neck squamous cell carcinoma. Oncol Lett 15:2259-2265. https://doi.org/10.3892/ol.2017.7564 
8. Cho YA, Yoon HJ, Lee Jl et al (2011) Relationship between the expressions of PD-L1 and tumor-infiltrating lymphocytes in oral squamous cell carcinoma. Oral Oncol 47:1148-1153.

https://doi.org/10.1016/j.oraloncology.2011.08.007

9. Cohen EEW, Soulières D, Le Tourneau C et al (2019) Pembrolizumab versus methotrexate, docetaxel, or cetuximab for recurrent or metastatic head-and-neck squamous cell carcinoma (KEYNOTE-040): a randomised, open-label, phase 3 study. Lancet 393:156-167. https://doi.org/10.1016/S01406736(18)31999-8

10. Duan XS, Lu J, Ge ZH et al (2013) Effects of T-cadherin expression on B16F10 melanoma cells. Oncol Lett 5:1205-1210. https://doi.org/10.3892/ol.2013.1164

11. Evrard D, Hourseau M, Couvelard A et al (2020) PD-L1 expression in the microenvironment and the response to checkpoint inhibitors in head and neck squamous cell carcinoma. Oncoimmunology 9:1844403. https://doi.org/10.1080/2162402X.2020.1844403

12. Ferlay J, Soerjomataram I, Dikshit R et al (2015) Cancer incidence and mortality worldwide: sources, methods and major patterns in GLOBOCAN 2012. Int J Cancer 136:E359-E386.

https://doi.org/10.1002/ijc.29210

13. Ferrand F, Malka D, Bourredjem A et al (2013) Impact of primary tumour resection on survival of patients with colorectal cancer and synchronous metastases treated by chemotherapy: results from the multicenter, randomised trial Federation Francophone de Cancerologie Digestive 9601. Eur J Cancer 49:90-97. https://doi.org/10.1016/j.ejca.2012.07.006

14. Hong AM, Ferguson P, Dodds T et al (2019) Significant association of PD-L1 expression with human papillomavirus positivity and its prognostic impact in oropharyngeal cancer. Oral Oncol 92:33-39. https://doi.org/10.1016/j.oraloncology.2019.03.012

15. Hong AM, Vilain RE, Romanes S et al (2016) PD-L1 expression in tonsillar cancer is associated with human papillomavirus positivity and improved survival: implications for anti-PD1 clinical trials. Oncotarget 7:77010-77020. https://doi.org/10.18632/oncotarget.12776

16. Huang ZY, Wu Y, Hedrick N et al (2003) T-cadherin-mediated cell growth regulation involves $\mathrm{G} 2$ phase arrest and requires p21(CIP1/WAF1)expression. Mol Cell Biol 23:566-578. https://doi.org/ 10.1128/MCB.23.2.566-578.2003

17. Kyriakakis E, Maslova K, Philippova M et al (2012) T-Cadherin is an auxiliary negative regulator of EGFR pathway activity in cutaneous squamous cell carcinoma: impact on cell motility. J Invest Dermatol 132:2275-2285. https://doi.org/10.1038/jid.2012.131

18. Leemans CR, Braakhuis BJ, Brakenhoff RH (2011) The molecular biology of head and neck cancer. Nat Rev Cancer 11: 9-22. https://doi.org/ 10.1038/nrc2982

19. Mukoyama Y, Zhou S, Miyachi Y et al (2005) T-cadherin negatively regulates the proliferation of cutaneous squamous carcinoma cells. J Invest Dermatol 124:833-838. https://doi.org/10.1111/j.0022202X.2005.23660.x

20. O’Rorke MA, Ellison MV, Murray LJ et al (2012) Human papillomavirus related head and neck cancer survival: a systematic review and meta-analysis. Oral Oncol 48:1191-1201. https://doi.org/10.1016/j.oraloncology.2012.06.019 
21. Ohigashi Y, Sho M, Yamada Y et al (2005) Clinical significance of programmed death-1 ligand-1 and programmed death-1 ligand-2 expression in human esophageal cancer. Clin Cancer Res 11:2947-2953. https://doi.org/10.1158/1078-0432.CCR-04-1469

22. Philippova M, Pfaff D, Kyriakakis E et al (2013) T-cadherin loss promotes experimental metastasis of squamous cell carcinoma. Eur J Cancer 49:2048-2058. https://doi.org/10.1016/j.ejca.2012.12.026

23. Schneider S, Kadletz L, Wiebringhaus R et al (2018) PD-1 and PD-L1 expression in HNSCC primary cancer and related lymph node metastasis impact on clinical outcome. Histopathology 73:573-584. https://doi.org/10.1111/his.13646

24. Seiwert TY, Burtness B, Mehra R et al (2016) Safety and clinical activity of pembrolizumab for treatment of recurrent or metastatic squamous cell carcinoma of the head and neck (KEYNOTE-012): an open-label, multicentre, phase 1b trial. Lancet Oncol 17:956-965. https://doi.org/10.1016/S1470-2045(16)30066-3

25. Ski Skinner HD, Giri U, Yang LP et al (2017) Integrative Analysis Identifies a Novel AXL-PI3 Kinase-PD-L1 Signaling Axis Associated with Radiation Resistance in Head and Neck Cancer. Clin Cancer Res 23:27132722. https://doi.org/10.1158/1078-0432.CCR-16-2586

26. Suda K, Rozeboom L, Rivard CJ et al (2017) Therapy-induced E-cadherin downregulation alters expression of programmed death ligand-1 in lung cancer cells. Lung Cancer 109:1-8. https://doi.org/ 10.1016/j.lungcan.2017.04.010

27. Sun $\mathrm{Y}$, Jing J, Xu $\mathrm{H}$ et al (2021) N-cadherin inhibitor creates a microenvironment that protect TILs from immune checkpoints and Treg cells. J Immunother Cancer 9:e002138. https://doi.org/ 10.1136/jitc-2020002138

28. Thompson RH, Gillett MD, Cheville JC et al (2004) Costimulatory B7-H1 in renal cell carcinoma patients: Indicator of tumor aggressiveness and potential therapeutic target. Proc Natl Acad Sci U S A 101:1717417179. https://doi.org/10.1073/pnas.0406351101

29. Ulivi P, Zoli W, Calistri D et al (2006) p16INK4A and CDH13 hypermethylation in tumor and serum of nonsmall cell lung cancer patients. J Cell Physiol 206:611-615. https://doi.org/10.1002/jcp.20503

30. Wang Q, Chen Y, Chen Y et al (2021) Aberrant promoter methylation of T-cadherin in sera is associated with a poor prognosis in oral squamous cell carcinoma. Neoplasma 68:528-534. https://doi.org/10.4149/neo_2021_201110N1203

31. Wang Q, Zhang X, Song X et al (2018) Overexpression of T-cadherin inhibits the proliferation of oral squamous cell carcinoma through the PI3K/AKT/mTOR intracellular signalling pathway. Arch Oral Biol 96:74-79. https://doi.org/10.1016/j.archoralbio.2018.08.018

32. Widschwendter A, Ivarsson L, Blassnig A et al (2004) CDH1 and CDH13 methylation in serum is an independent prognostic marker in cervical cancer patients. Int J Cancer 109:163-166. https://doi.org/10.1002/ijc.11706

33. Zhou AL, Wang X, Yu W et al (2020) Expression level of PD-L1 is involved in ALDH1A1-mediated poor prognosis in patients with head and neck squamous cell carcinoma. Pathol Res Pract 216:153093. https://doi.org/10.1016/j.prp.2020.153093

\section{Figures}



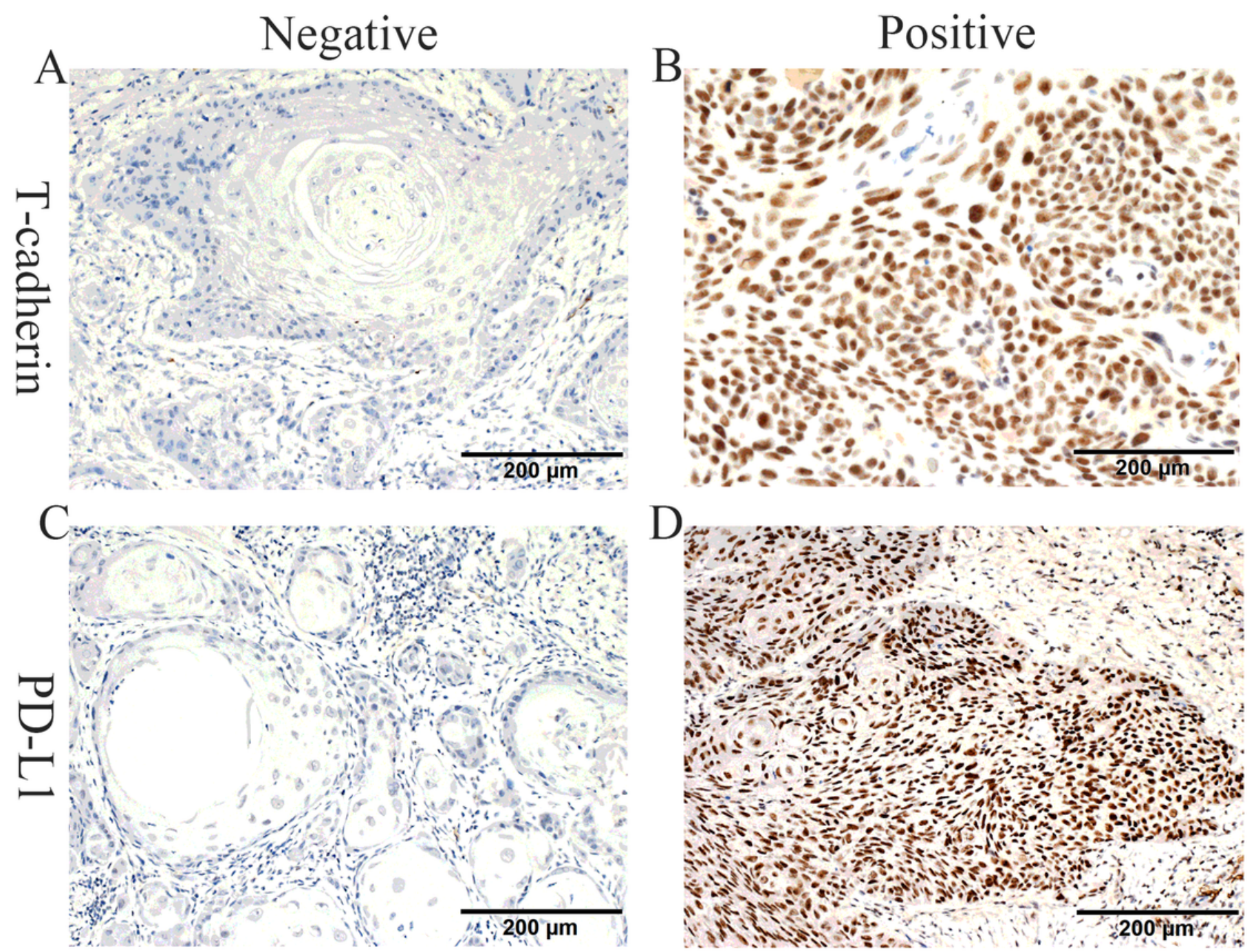

\section{Figure 1}

Immunohistochemical staining of T-cadherin and PD-L1 in representative HPV negative head and neck squamous cell carcinoma tissues. (A) T-cadherin negative; (B) T-cadherin positive; (C) PD-L1 negative; (D) PDL1 positive. (all magnification, x400). PD-L1, programmed cell death 1 ligand 1. 


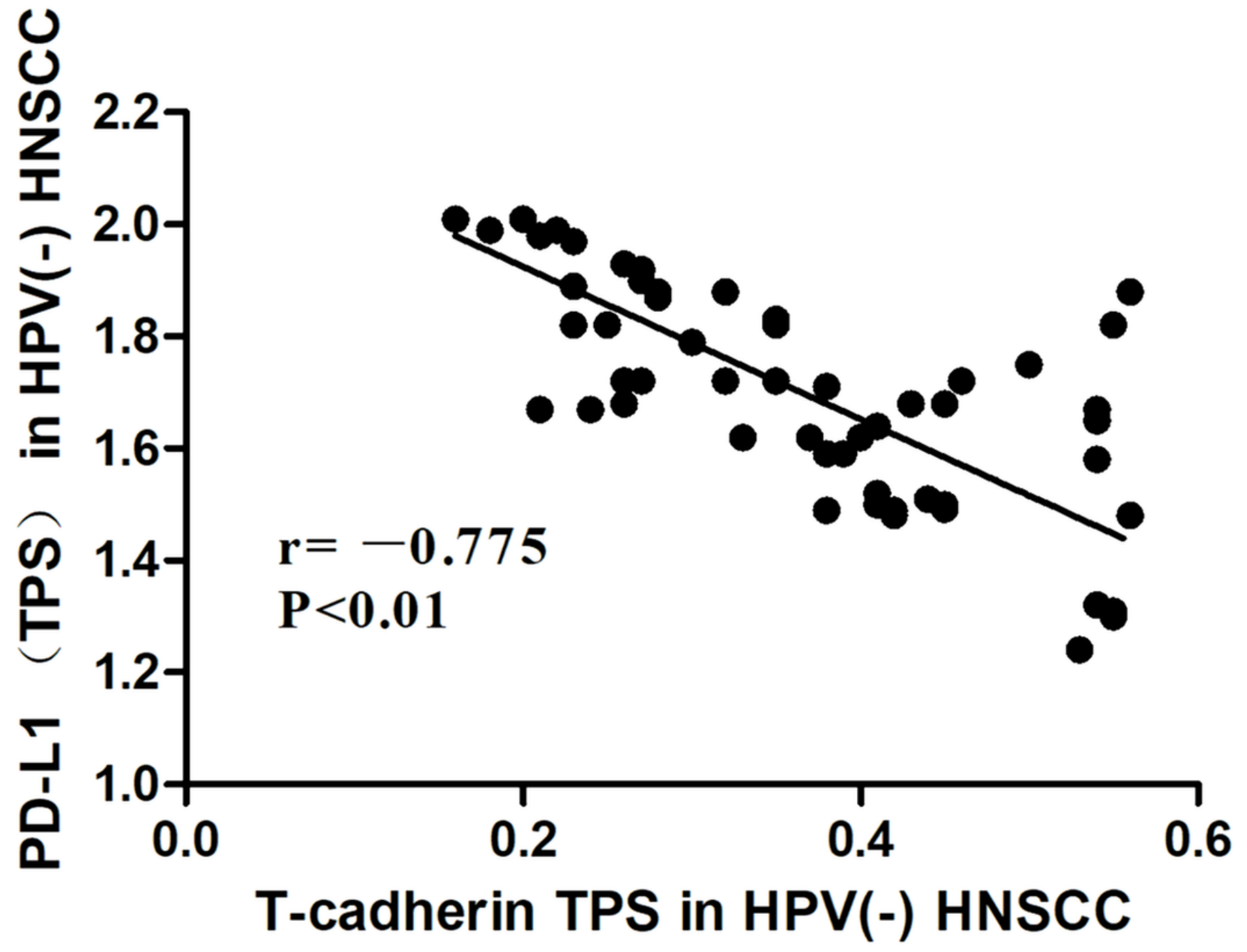

Figure 2

Correlation between T-cadherin protein expression and PD-L1 protein expression. TPS, tumor proportion score. 

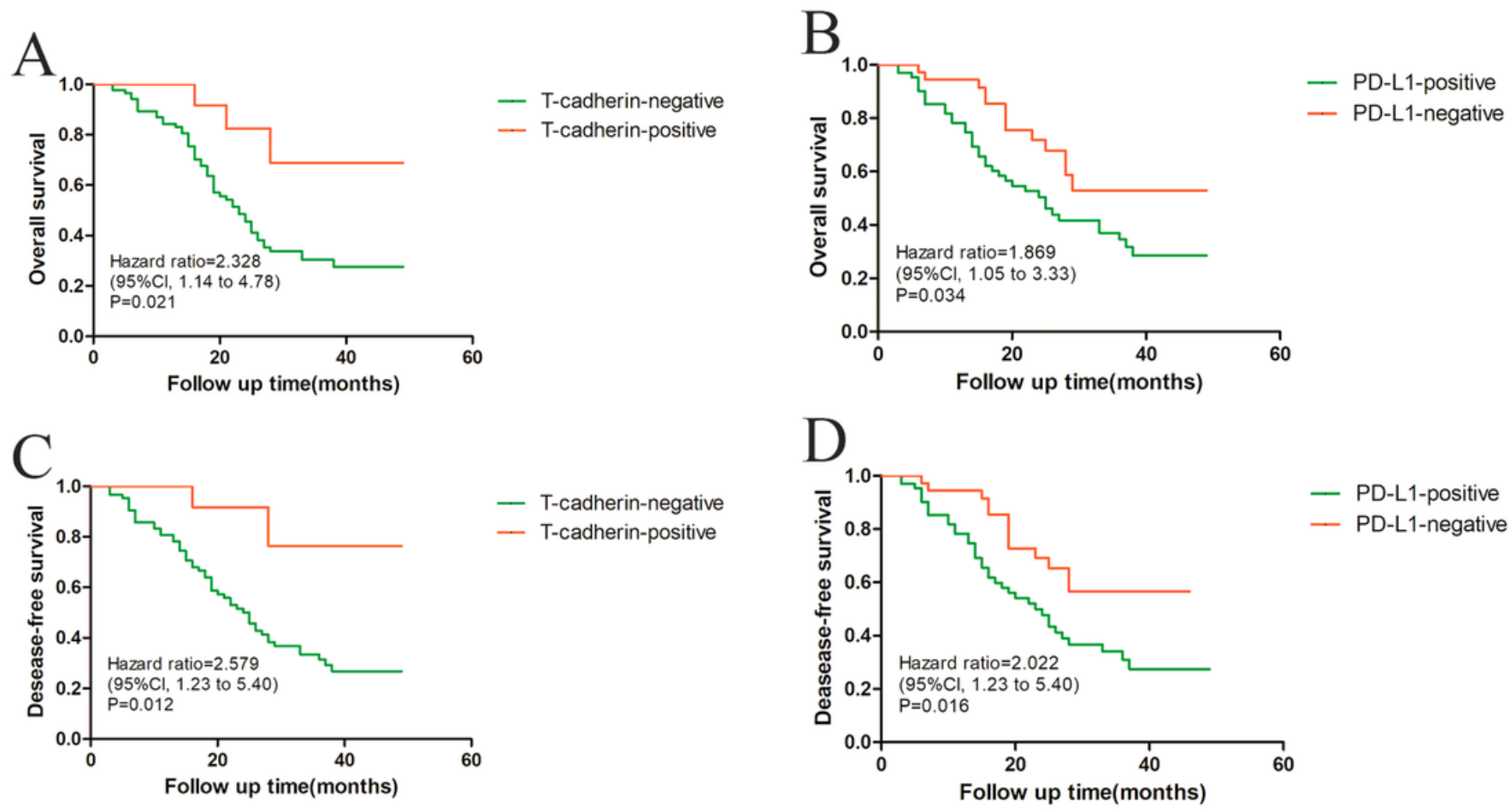

\section{Figure 3}

Kaplan-Meier curves for patients with head and neck squamous cell carcinoma. Overall survival and deseasefree survival of patients with high vs low T-cadherin (A\&C) and PD-L1 (B\&D) protein expression. 


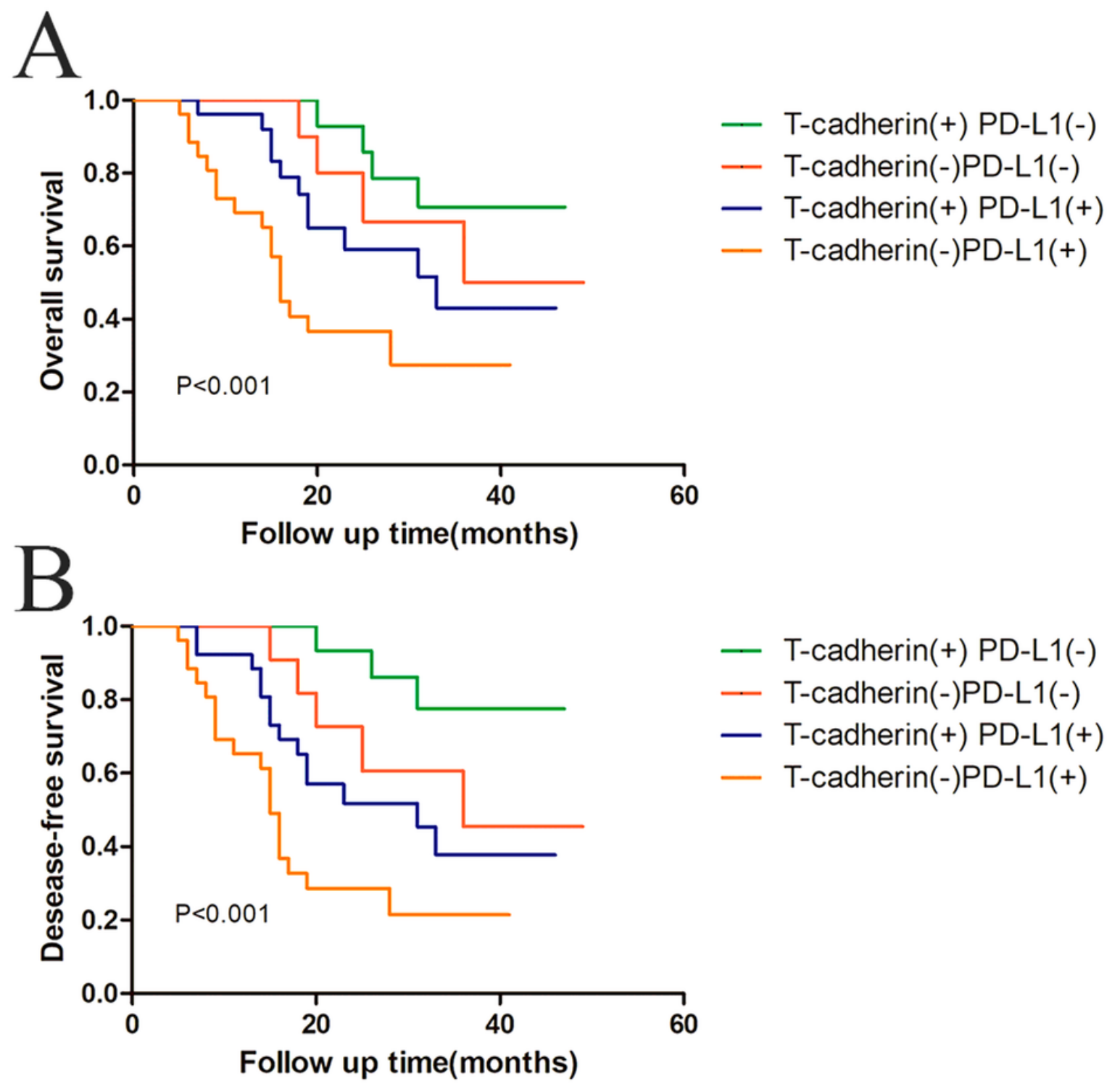

Figure 4

Combination analysis of the effects of T-cadherin and PD-L1 on overall survival (A) and desease-free survival (B) of patients with head and neck squamous cell carcinoma. 